\title{
Determination of Time Interval between Hospitalization and Establishment of Naso Jejunal Feeds in Acute Pancreatitis- A Western Indian Study
}

\author{
Deshpande Aa ${ }^{1}$, Shah $\mathrm{Rk}^{2}$, Raskar Aa ${ }^{2}$, Shukla Kk ${ }^{1}$, Awale Pk. ${ }^{2}$ \\ ${ }^{1}$ Department of Surgery, Seth G.S. Medical College and K.E.M. Hospital, Mumbai, India \\ ${ }^{2}$ Department of Infectious Diseases, Seth G.S. Medical College and K.E.M. Hospital, Mumbai, India
}

\begin{abstract}
Acute Pancreatitis (AP) is characterized by diffuse inflammatory process of pancreas with variable involvement of adjacent tissues and dysfunction of remote organs. Mortality rate in patients with AP is reported in the range of 9\%-27\% with two peaks. Nutritional support is one of the key factors in improving outcomes in cases of AP as per the current guidelines. Several clinical studies and meta-analysis regarding the effectiveness of enteral nutrition conclude that enteral nutrition through nasojejunal route is safe and can preserve the integrity of intestinal mucosa to decrease the incidence of infectious complications and other severe complications as well as resulted in better control of blood glucose levels. Enteral nutrition as early as within 24 hours or 48 hours has been recommended in various clinical studies. Though early enteral nutrition has been shown to reduce infectious complications in western literature, in clinical practice, it is not always possible to institute feeds so early. There can be delay due to factors like patient factors and extrinsic factors. While patient factors can include prolonged ileus, patients with altered mental condition patients on ventilatory support etc, extrinsic factors can be non-working of machine, other logistical problems and non-tolerance of feeds. We intended to determine the average time interval between the hospitalization of patients and establishment of feeds in our patients of AP. This was an open label longitudinal study conducted in a single surgical unit at a tertiary referral hospital in Mumbai. The sample size was 61 and study was conducted for a period of 6 months. For statistical analysis, descriptive statistics was used. To find average time, arithmetic mean was used. The average time interval is found to be 4.6 days.
\end{abstract}

Keywords: Acute pancreatitis, enteral nutrition, nasojejunal feeds.

\section{Introduction}

Acute Pancreatitis (AP) is one of the most common pancreatic diseases and is characterized by diffuse inflammatory process of pancreas with variable involvement of adjacent tissues and dysfunction of remote organs. The clinical severity varies from mild pancreatitis to severe necrotizing pancreatitis [1]. In $20 \%$ cases, AP is severe with or without pancreatic necrosis. These patients have a much higher morbidity and mortality. The current recommendation for treatment of pancreatitis emphasises role of early resuscitation, aggressive monitoring, and early nutritional support.[2] In severe acute pancreatitis,(SAP), enteral feeding has been found superior to parenteral nutrition due to its beneficial effects on the gut barrier [3]. Several clinical studies and meta-analysis regarding the effectiveness of enteral nutrition have proven safety of enteral nutrition and has also shown to preserve the integrity of intestinal mucosa to decrease the incidence of infectious complications and hospital stay.[4] A meta-analysis of 11 studies claims that institution of enteral feeds early within 48 hours reduces infectious complications. [5] Enteral route is more convenient in clinical practice. Enteral nutrition can be given by nasogastric or nasojejunal route,[6] both having their advantages and disadvantages. In our hospital the naso jejunal route is used as a preferred route of enteral nutrition. Insertion of a nasojejunal tube requires radiologic or endoscopic guidance. Though some studies have instituted enteral feeding as early as within 24 hours,[3], in clinical practice we find in our patients that feeds cannot be instituted so early. Sometimes there can be delay in instituting feeds due to patient factors like prolonged ileus, patients with disoriented mental condition or patients on ventilatory support or extrinsic factors like non- working of machine, non-tolerance of feeds or others. We undertook this study to determine the average time interval between the hospitalization of patients and establishment of nasojejunal feeds in the management of our patients with acute pancreatitis. The average time for recovery from ileus and actual implementation of nasojejunal feeds was studied. We also studied the rate of successful institution of nasojejunal feeds.

\section{Materials and methods}

Study was conducted in compliance with the protocol and regulatory requirements. This was an open label, observational longitudinal study. Study was carried out at in a single surgical unit in a tertiary referral centre. Approval of Institutinal Ethics committee was taken prior to initiation of study. Waiver of taking 
informed consent was granted by ethics committee. The Study cohort included all patients admitted with a diagnosis of acute pancreatitis in a single surgical unit over a period of 6 months.

All the patients who required ventilatory support during the course of illness and those in whom there was loss of data due to patient taking discharge against medical advice were excluded. In all 70 patients were enrolled for the study. Nine were excluded for analysis. For clinical severity, the Ranson's score and APACHE II score entered in the patients' papers were noted. Also the Computerised Tomography (CT) severity index (CTSI) as per the CT scan report was recorded. Duration between hospital admission to recovery from ileus as well as starting of enteral feeds was noted. Also the failure to tolerate enteral nutrition if any was recorded. For statistical analysis descriptive statistics was used. To calculate the average time interval between hospitalization of patients to recovery from ileus as well as starting of enteral feeds, arithmetic mean was used.

\section{Results}

In this study, patients with diagnosed acute pancreatitis were enrolled as per inclusion and exclusion criteria. In all 70 patients were enrolled in the study of which 18 patients were females and 52 patients were males. The average age of patients including both the sexes was 35 years. Nine patients were excluded from analysis.. Average Apache II score and Average CT severity index was found to be 6.77 and 7.22 respectively. For calculating the average time interval descriptive statistics was used. Average time interval between hospitalization and recovery from ileus was 4.2 days. Average time interval between hospitalization and establishment of enteral nutrition was found to be 4.6 days. There was a delay of 1 day between the clinician's opinion about the patient's readiness for institution of enteral feeds and actual day of institution of enteral feeds. During the course of the study, no severe outcomes were reported. In 18/61 number of cases, there was failure of insertion of tube or failure to tolerate feeds. Successful implementation of nasojejunal feeds was carried out in $43 / 61$ patients.

Graph 1. Apache II scores of patients

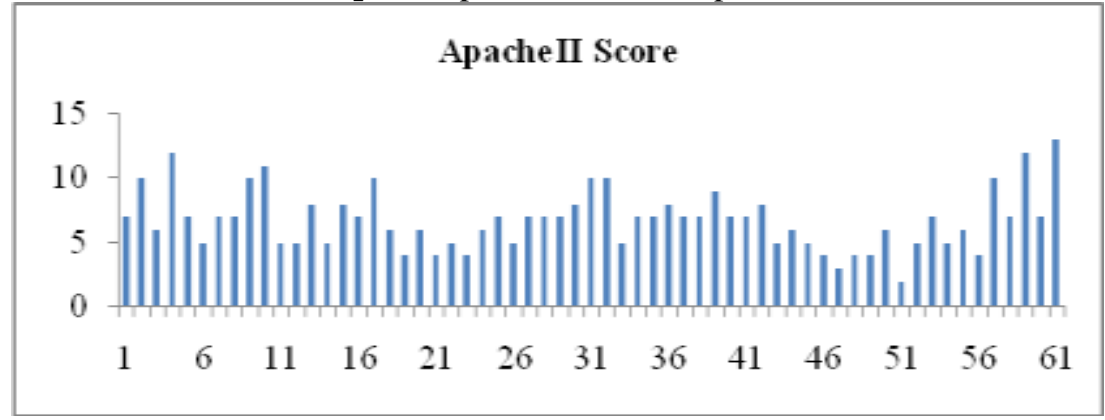

Graph 2. CT severity index of patients

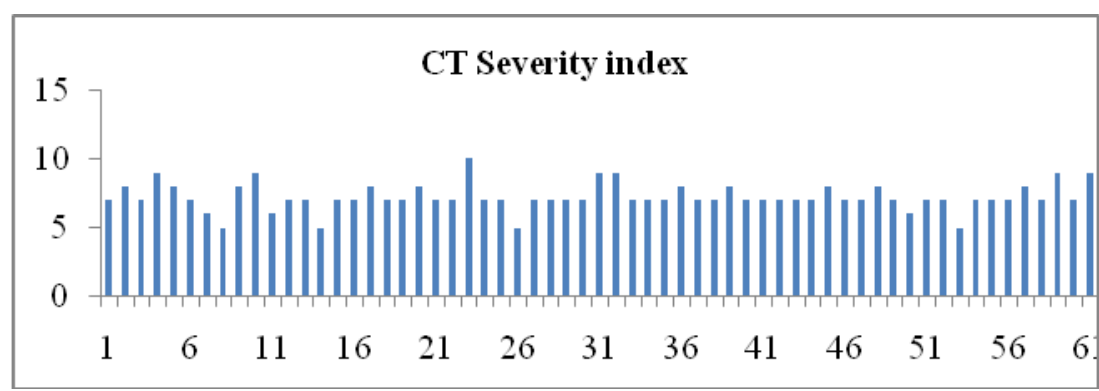

Graph 3. Interval between hospitalization and recovery from paralytic ileus

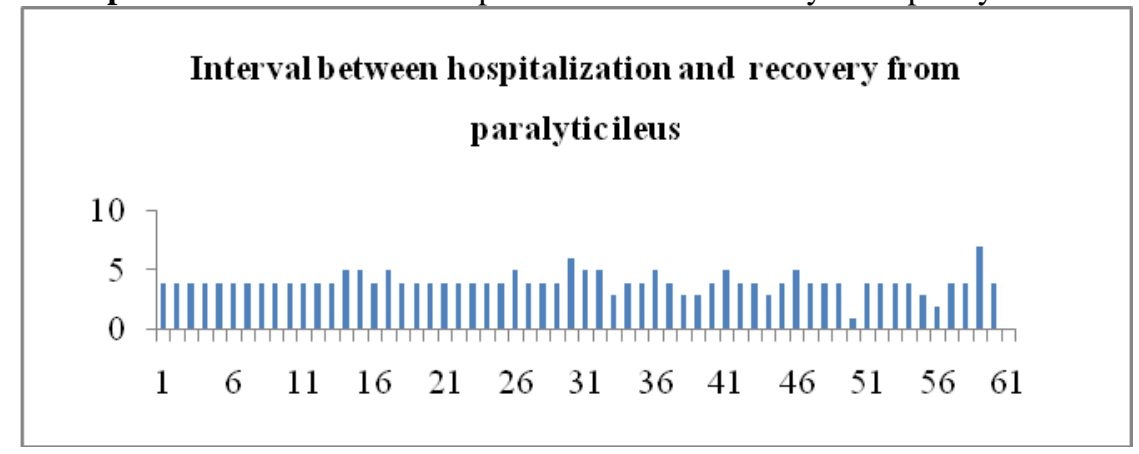


Graph 4. Interval between actual implementation of nasojejunal feeds after .

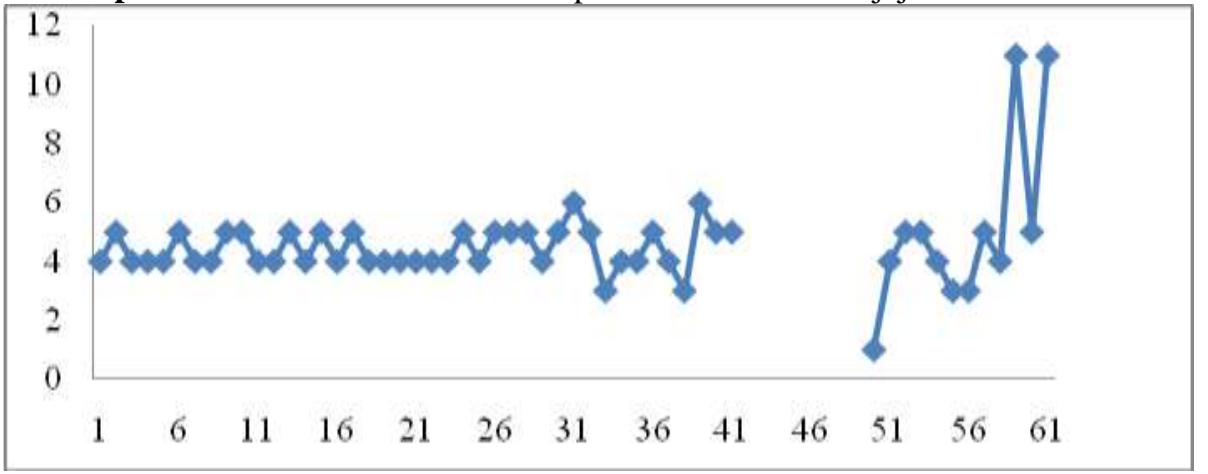

Graph 5. Rate of successful institution of nasojejunal enteral nutrition

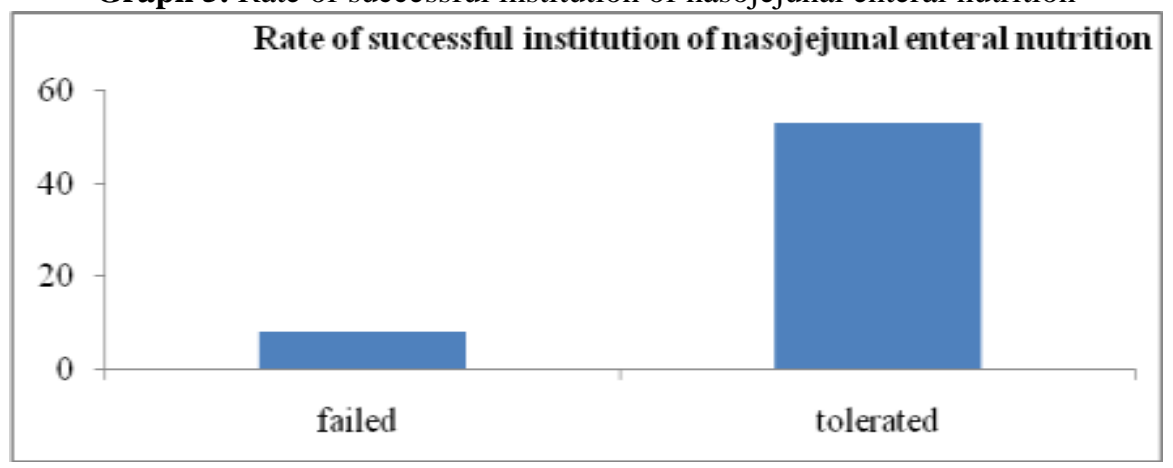

\section{Discussion}

Acute pancreatitis continues to be a serious illness, and the patients with acute pancreatitis are at risk to develop different complications from ongoing pancreatic inflammation. In the first week, severe AP is associated with Systemic Inflammatory Response Syndrome (SIRS). In this, there is hypermetabolism and high protein catabolism. This causes acute malnutrition in these patients and increased calorie requirement. Acute malnutrition leads to impaired immune function, increased risk of sepsis, poor wound healing, and multiple organ failure(MOF).[7] After the second week there can be complications of pancreatic necrosis especially infection, which further increases the energy requirement. Thus, nutrition management is now recognised as an important factor in treating these patients.[8]

Oral or enteral feeding used to be thought harmful in cases of AP due to stimulation of exocrine pancreatic function and autodigestion. As a consequence, total parenteral nutrition (TPN) and bowel rest were routine recommendations in management of AP. [9]. However, many studies recognised the importance of intergrity of gut barrier to prevent bacterial translocation and also to maintain immune function.[10] Also, enteral nutrition given in jejunum does not stimulate the pancreatic exocrine function. Thus, TPN and jejunal feeding became the mainstay of nutritional management in AP.[8]

Recently there have been many studies using nasogastric (NG) feeds instead of nasojejunal (NJ) feeds in AP. $[3,8]$ This concept is basically contradictory to that of pancreatic rest. Also, presence of gastric ileus may lead to failure of NG feeding and also lead to possibility of aspiration pneumonia. The only advantage is that the NG tube can be positioned in the ward without any special techniques. In our institution, we prefer the nasojejunal route of feeding. $\mathrm{NJ}$ requires expertise of endoscopist or radiologist for tube insertion.

The timing of institution of enteral feeds differ from very early, (within 24 hours) to upto 4 days after hospitalisation in various studies.[3,11] Though institution of feeds before 48 hours of onset has been associated with reduction in morbidity and infectious complications[5], in clinical practice, we find that it is not possible to initiate feeds so early in all patients. Recovery from ileus differs in all patients. It will also depend upon the local inflammation. In the study group, average APACHE II score of patients was 6.7. These patients had low rate of systemic complications. However, the average CT severity index was found to be 7.22 which implies that the local pancreatic inflammation was more severe. These patients thus belong to the moderately severe category.[12] These patients will take longer to recover from ileus. Also there are other extrinsic factors which can delay the insertion of nasojejunal intubation. Our study focuses on the average interval to establishment of enteral feeds in patients with acute pancreatitis. The average time interval from hospitalization to recovery from paralytic ileus was found to be 4.2 days and the average time interval between hospitalization and establishment of enteral nutrition was 4.6 days. Due to logistical factors other than patient factors, there is an additional delay 
of 0.4 days and the time interval for actual implementation of nasojejunal feeds after hospitalization was 4.6 days. Thus, there is considerable time gap before complete caloric supplementation can be implemented by enteral route. In $87 \%$ of patients nasojejunal feeds were successfully implemented. The failure rate of test feeds was $8 / 61$ patients $(13 \%)$. There were no bad outcomes in the study group. Whether total parenteral nutrition is necessary during the initial phase of inadequate caloric requirement, can be a further research question.

Drawbacks of this study include that patients with very high severity were not included in the analysis. Also this study can be further substantiated by larger clinical studies including larger number of patients and multiple parameters.

\section{Conclusion}

Nasojejunal feeds can be instituted in most patients of AP. The success rate of enteral nutrition is more than $85 \%$. The delay between hospitalisation and institution of feeds is more than 4 days. Largely our patients belong to the category of moderately severe pancreatitis and hence probably have a longer period of ileus.

\section{Acknowledgement}

We wish to acknowledge Dr. A. N. Supe, Director (ME \& MH) and Dean, Seth G. S. Medical College $\&$ K.E.M. Hospital, for permitting us to publish hospital data.

\section{References}

[1]. Harper SJ, Cheslyn-Curtis S. Acute pancreatitis. Ann Clin Biochem. 2011 Jan;48(1):23-37.

[2]. Tenner S, Baillie J, DeWitt J, Vege SS. American College of Gastroenterology guideline: management of acute pancreatitis. Am J Gastroenterol. 2013 Sep;108(9):1400-15; 1416

[3]. Eckerwall GE, Axelsson JB, Andersson RG. Early nasogastric feeding in predicted severe acute pancreatitis: A clinical, randomized study. Ann Surg. 2006 Dec;244(6):959-65; discussion 965-7.

[4]. Paul E. Marik, Gary P. Zaloga. Meta analysis of parenteral nutrition versus enteral nutrition in patients with acute pancreatitis. BMJ, June 2004

[5]. Jie-Yao Li, Tao Yu, Guang-Cheng Chen, Yu-Hong Yuan, Wa Zhong, Li-Na Zhao, Qi-Kui Chen. Enteral Nutrition within 48 Hours of Admission Improves Clinical Outcomes of Acute Pancreatitis by Reducing Complications: A Meta-Analysis. PLoS One. 2013; 8(6): e64926.

[6]. Doley RP, Yadav TD, Wig JD, et al. Enteral Nutrition in Severe Acute Pancreatitis. JOP. J Pancreas 2009 Mar 9; $10(2)$ : $157-62$.

[7]. Abou-Assi S, O'Keefe SJ. Nutrition in acute pancreatitis. J Clin Gastroenterol. 2001;32:203-209.

[8]. Kun Jiang, Xin-Zu Chen, Qing Xia, Wen-Fu Tang, Lei Wang. Early nasogastric enteral nutrition for severe acute pancreatitis. World J of gastroenterol. 2007 Oct 21; 13 (39): 5253-60.

[9]. Meier R, Beglinger C, Layer P, Gullo L, Keim V, Laugier R, Friess H, Schweitzer M, Macfie J. ESPEN guidelines on nutrition in acute pancreatitis. European Society of Parenteral and Enteral Nutrition. Clin Nutr. 2002;21:173-83.

[10]. Hadfield RJ, Sinclair DG, Houldsworth PE, Evans TW. Effects of enteral and parenteral nutrition on gut mucosal permeability in the critically ill. Am J Respir Crit Care Med. 1995;152:1545-8.

[11]. Piciucchi M, Merola E, Marignani M, Signoretti M, Valente R, Cocomello L, Baccini F, Panzuto F, Capurso G, Delle Fave G. Nasogastric or nasointestinal feeding in severe acute pancreatitis. World J Gastroenterol. 2010 Aug 7;16(29):3692-6.

[12]. Vege SS, Gardner TB, Chari ST, Munukuti P, Pearson RK, Clain JE, Petersen BT, Baron TH, Farnell MB, Sarr MG. Low mortality and high morbidity in severe acute pancreatitis without organ failure: a case for revising the Atlanta classification to include "moderately severe acute pancreatitis". Am J Gastroenterol. 2009 Mar;104(3):710-5. 